\title{
La vinculación con la sociedad en el Ecuador: Reflexiones sobre su itinerario curricular
}

\author{
Higher education outreach and engagement in Ecuador: Reflexions about their curriculum itinerary
}

\section{Héctor Alfonso Simbaña Cabrera ${ }^{(* a)}$, Lorena Elizabeth Correa $^{(b)}$}

(a*) Dirección General de Vinculación con la Sociedad, Universidad Central del Ecuador, Quito, Ecuador. hasimbana@uce.edu.ec (b) Ayudante de cátedra.

\section{Resumen}

La extensión universitaria en las universidades del Ecuador, históricamente, se han encontrado atadas a las vicisitudes políticas, económicas y sociales del país, y su desarrollo, en una u otra circunstancia, ha impulsado el avance o retroceso de la academia. Este es un reto permanente para los actores universitarios, toda vez que el avance incontenible de la ciencia y la tecnología demanda de la casona de estudios mayores niveles de calidad y pertinencia en la formación de profesionales. Potencializar su trascendencia académica y política, exige a la universidad actual, analizar sus experiencias en este campo, contextualizarlas a la realidad de los pueblos de Ecuador, para proyectarlas en el siglo XXI, desde una perspectiva trasformadora, holística e integral, como instrumento que garantice un cambio cualitativo en la educación superior. Este cambio necesariamente exige diseñar un nuevo proyecto educativo, que tome en cuenta a la vinculación con la sociedad o extensión universitaria como el eje de desarrollo académico, en el cual confluyan de forma dialéctica las funciones sustantivas de investigación, docencia y extensión.

Palabras clave: extensión universitaria, vinculación con la sociedad, sociedad.

\section{Abstract}

Historically, the function of the Universities of Ecuador called "Extension Universitaria" has been tied to the political, economic, and social difficulties of the country. Its development in various situations has encouraged progress or regression of the academy, becoming a permanent challenge for University actors, since the unstoppable advances of science and technology demand higher levels of quality and relevance in trained professionals. Because of its academic and political significance, the university is required to analyze their experiences in this field, contextualize the reality of Ecuadorian people, and project them into the twenty-first century, all from a transformative, holistic, and comprehensive perspective as an instrument aiming to ensure a qualitative change in Higher Education. This change requires designing a new educational project that takes into account universities' relationship with society or "Extension Universitaria" as the axis of the academic development, which aims to bring together the substantive functions of research, teaching, and extension.

Key words: university extension, society outreach, society. 


\section{Introducción}

Desde los primeros albores de la universidad europea del siglo XVIII, la extensión universitaria ha recorrido un importante camino de transformación que ha dado como resultado un sinnúmero de modelos, concepciones e innovaciones de estudio en permanente relación con los diversos contextos sociales e intereses que se ciernen sobre las instituciones de educación superior.

En América Latina, la extensión universitaria, hoy denominada en el Ecuador, Vinculación con la Sociedad, surge irreverente con el movimiento de Córdova (Argentina) mediante la Reforma Universitaria a inicios del siglo XX. Rápidamente se convirtió en una proclama de la juventud, que trasciende a todos los países del continente, por la contundencia de sus planteamientos, de los cuales sobresalen la autonomía universitaria, la libertad de cátedra y la extensión universitaria, en particular.

Este planteamiento universitario, en función del desarrollo social, se irá consolidando como uno de los pilares fundamentales del quehacer en medio de una profunda e intensa agitación social, que caracteriza a todos los países de América Latina, producto de la confrontación entre las ideas progresistas versus las conservadoras de la sociedad de la época y que, necesariamente, se expresa en el claustro universitario.

En este escenario, la extensión universitaria, nace fertilizada y alimentada de las ideas humanistas de corte marxista, fundamentadas en la dialéctica de la naturaleza, como teoría científica, que explica la esencia de todos los fenómenos sociales y políticos y que, por supuesto, incluye al fenómeno educativo y la universidad, como parte del andamiaje social.

En el Ecuador, los principios universitarios como la autonomía universitaria, solo serán reconocidos a partir de 1940, más de una década de constante exigencia de los sectores sociales organizados y los no organizados. De esta manera, la vieja casona abandona su estructura medieval y escolástica, en procura de responder a las necesidades de profesionalización de cientos de jóvenes de una sociedad extremadamente polarizada entre ricos y pobres, en el marco de la implantación del proceso productivo, calificado por los historiadores como el desarrollismo, impulsado por los gobiernos de la época.
La autonomía universitaria abrirá un espacio de participación en las aulas universitarias a las clases sociales emergentes y que consolidarán esta exigencia social, a partir de la segunda mitad del siglo XX. La Federación de Estudiantes Universitarios del Ecuador (FEUE) y de los docentes son los principales protagonistas y promotores de la reforma universitaria y de la extensión universitaria, como planteamiento fundamental en lo que vendría a ser una propuesta seria de trasformación de la educación universitaria.

Inicia de esta forma un permanente proceso en el que la extensión universitaria, considerada como instrumento de conciencia social, convicción y voluntariado, se instala en la institución de educación superior más antigua del Ecuador, como metodología de trabajo. Su objetivo final se manifiesta en la necesidad de integrar a los estamentos universitarios a través de proyectos comunitarios interdisciplinarios, con la participación de importantes sectores populares durante las décadas de los años 60 y 70, especialmente en las grandes ciudades como Guayaquil, Quito y Cuenca.

La toma del Comité del Pueblo, el Centro Histórico y algunos barrios populares en la capital de la república, Quito, sobresalen como muestra de potencial académico y social de la extensión universitaria, en la cual se integran actores comunitarios con elementos universitarios, para enfrentar la problemática identificada en las áreas de vivienda, salud, educación y organización social, reconocida a nivel nacional e internacional; que sentará las bases para constituir propuestas educativas innovadoras en los siguientes años, en las Facultades de Jurisprudencia, Filosofía, Medicina, Ingeniería, Arquitectura. Así, se evidencia importantes avances curriculares y sociales mediante los Consultorios Jurídicos Populares, el Departamento de Ingeniería Popular, el Taller de Investigación Social Diseño y Comunicación (TISDYC) y los programas de salud y educación, entre otros.

Y si bien los logros sociales fueron relevantes, al interior del recinto universitario, la praxis comunitaria, impregnada en la conciencia de los actores, impulsó un hecho subjetivo por demás trascendente, los ideales de la Segunda Reforma Universitaria, que colocó, al centro del debate la concepción misma de la universidad y de la educación en la sociedad ecuatoriana, que finalmente se expresó en la lucha por la conquista de la gratuidad de la educación y del cogobierno universitario. 
A pesar de estas importantes experiencias y luego de un sinuoso devenir histórico, los años 1980 y 90 serán en los que se produce una inflexión de la extensión universitaria. Ya incorporada de manera formal en la normativa legal o estatutaria, del país y de la universidad, no logró constituirse en una política institucionalizada, como parte del currículo universitario y en la que necesariamente participe toda la universidad.

De hecho el balance del organismo rector de las universidades en el Ecuador, el conocido en ese entonces como El Consejo Nacional de Universidades y Escuelas Politécnicas (CONUEP), en el año 1992, sostiene, que existe un desfase entre la universidad ecuatoriana y la sociedad nacional en la que se desenvuelve. Mientras que el país requiere con verdadera urgencia el avance científico y tecnológico, las instituciones mantienen un esquema de facultades profesionalizantes. Mientras se precisa carreras técnicas especializadas, la universidad tiende a seguir impartiendo una formación enciclopédica y generalista (Hallo, 2009 y Wilson, 2012).

Por lo expuesto, se evidencia que la extensión universitaria, por lo menos en la Universidad Central del Ecuador, no superó los esfuerzos de los estudiantes y de un sector de los docentes, quienes de forma aislada en las facultades de Arquitectura y de Filosofía, a fines de la década de los 90 e inicios del 2000, sostenían importantes programas comunitarios, mediante el TISDYC o los proyectos en educación, a través del Instituto de Extensión Universitaria (ISEU). Mientras en el conjunto de las facultades y de las universidades del país, se reduce a la mínima expresión de voluntariado o iniciativa de docentes y de estudiantes. Con ello las prácticas extensionistas desnaturalizan la esencia misma de la extensión universitaria.

El advenimiento del siglo XXI, caracterizado por la implementación de las políticas neoliberales en el ámbito educativo, marcó en el Ecuador el ascenso de la lucha social de los pueblos y de la juventud en particular. Un aspecto relevante es la exigencia de una educación gratuita, democrática, científica y transformadora, como eje sustancial para alcanzar cambios sociales significativos.

Estos aspectos determinarán un proceso intenso de agitación social en el ejercicio de cinco gobiernos consecutivos y que finalmente se verán materializados a partir de la aprobación de una nueva Constitución de la República en el año 2008, la Ley Orgánica de Educación Superior, (LOES) como instrumentos legales que promueven importantes avances conceptuales y que constituyen un substancial impulso para la ejecución de la Vinculación con la Sociedad, en el Reglamento de Régimen Académico y demás tratados legales vigentes en nuestro país.

Resulta evidente que con la vigencia de este marco legal, la Vinculación con la Sociedad en nuestro país se revitaliza y se potencializa en el quehace universitario a nivel nacional, como una de sus funciones llamadas a cumplir un rol preponderante en la formación de los profesionales en el Ecuador, aunque el debate de sus trascendencia, implementación, enfoques, modelos o proyectos demandan aún de una profundización que supera los aspectos normativos.

\subsection{La extensión universitaria es en esencia un debate de ideas}

El cuestionamiento al rol que cumple la universidad, desde las diversas esferas de la sociedad, de manera constante nos convoca a un análisis exhaustivo del tríptico misional en que se distingue a la DOCENCIA, como la función pedagógica, o proceso de enseñanza aprendizaje en el objetivo de formar profesionales, sustentados en la INVESTIGACIÓN CIENTÍFICA, como herramienta académica que le permite nutrirse, alimentarse de los insumos sociales y culturales mediante la función de LA EXTENSIÓN UNIVERSITARIA a modo de escenarios de aprendizaje que permite validar los conocimientos científicos desde la praxis social.

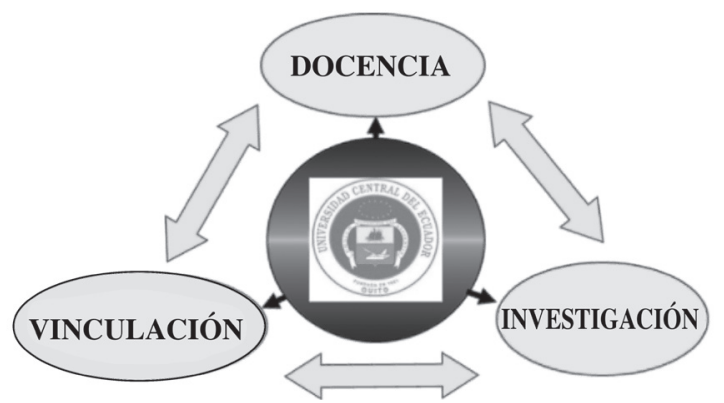

Figura 1: Tríptico Misional de la Universidad Central del Ecuador, por Sistema de Vinculación UCE (2016)

Se tomará a la Investigación, Docencia y Extensión, como unidad dialéctica, responsable de la formación de los profesionales y, por lo tanto, responsables de su calidad y pertinencia. El parámetro fundamental son las necesidades de 
desarrollo científico y social, en un escenario temporal y espacial con características determinadas.

En este ejercicio de responsabilidad académica, dicho cuestionamiento demanda para su realización, un posicionamiento filosófico y político en el que medían corrientes y tendencias del pensamiento sociológico, pedagógico y curricular, que permita edificar un proyecto educativo, a través del cual.

"La credibilidad y pertinencia de la Universidad del siglo XXI, se harán evidentes en la medida en que forjen profesionales cuya formación humanista y su alta capacitación científico - técnica sean fuentes constantes para la búsqueda de soluciones. El tríptico misional de la Universidad: formación, investigación y extensión, debe satisfacer y dar respuesta a las necesidades de la sociedad así como a ella misma (Batista de los Ríos, 2011, p152-161)".

Por tanto, la responsabilidad es compartida ya que la investigación y la docencia son pilares poseedores de un abundante fundamento teórico y técnico para su estudio y aplicación. En iguales circunstancias, es necesario reconocer que la función extensión universitaria también cuenta y es portadora de una epistemología y un sustento filosófico - teórico, que se orienta, en función de las concepciones o intereses que primen en su estudio y o aplicación.

Desde esta perspectiva, se puede entender el anuncio de un nuevo estadio de la extensión universitaria, como el resultado de un proceso sistémico realizado en los últimos tiempos en nuestro país, donde se afirma que la función a la que hacemos referencia, ha dejado de ser tal cosa para evolucionar a un aspecto más avanzado denominado Vinculación con la Sociedad.

En su defensa, se esgrimen argumentos que van desde los aspectos semánticos, hasta la posibilidad de superar las experiencias negativas del pasado. Pero se ha olvidado lo que hemos expuesto líneas arriba; la riqueza histórica, el contenido filosófico y epistemológico de la extensión universitaria, con lo que llegaríamos a la conclusión de que, la etiqueta que coloquemos a dicha función es totalmente relativa e intrascendente. Lo importante y hasta cierto punto determinante es el enfoque teórico que orientan dichos procesos.
Y lo que para algunos resulta ser fases evolutivas, para nosotros, en realidad, son enfoques, que se presentan en cualquier momento de la historia, como resultado de las nociones teóricas e ideológicas que orientan el quehacer y que se pueden describir como:

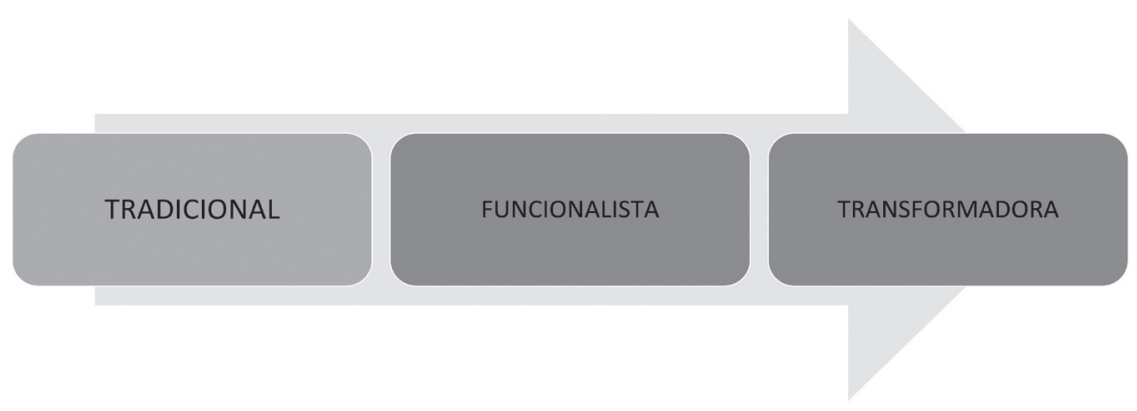

Figura 2: Enfoques de la Extensión Universitaria, por Héctor Simbaña (2016)

Tradicional: Enfoque cuya matriz generadora que se sustenta en la falta de compromiso o de conocimiento de una u otra actividad, sus manifestaciones son tan variadas como variados son los niveles de conocimiento y asimilación de los seres humanos. Sin embargo, la forma más sencilla de materialización se expresa en el asistencialismo.

El parámetro fundamental constituye la asistencia, lo importante de esta manifestación es "dar" o "servir" desde una visión unilateral, de superioridad que permanentemente termina en el activismo académico o institucional, carente de planificación y por tanto de impacto, en el que la calificación y la norma es lo importante.

Funcionalismo o Formalismo: Para algunos, esta forma resulta ser una fase o nivel, criterio con el que diferimos, pues creemos que en realidad se trata de una manifestación del enfoque tradicional, en la que la actividad puede incluso llegar a presentar trascendencia. Sin embargo, sus programas, proyectos y objetivos se encuentran preestablecidos, constituyen, por tanto, una acción burocrática, ajustada a las necesidades de quienes toman decisiones, generalmente en las instancias de poder. En este enfoque, lo más importante es la normativa o los aspectos legales necesarios de cumplir. 
Trasformador: Desde nuestra experiencia, la extensión universitaria o vinculación con la sociedad desde sus orígenes ha sido portadora de una esencia transformadora. Con características democráticas y participativas, fundamentado en la conciencia social, como proceso de autorrealización consciente, horizontal, en el que es posible un intercambio de saberes.

En este escenario, consecuentes con un enfoque trasformador y desde una perspectiva emancipadora, los aspectos de carácter normativo o legales no constituyen la principal preocupación, pues se entenderá que la verdadera trascendencia de dicha función sustantiva, insistimos, descansa en los principios conceptuales y filosóficos (ideas) y objetivos que orientan a esta función.

Entonces, como función social inherente al ser humano, resulta indispensable situar a la vinculación con la sociedad "en la corriente del humanismo marxista, que parte de la comprensión del hombre concreto, donde la transformación de las relaciones sociales constituyen la base para la lucha en su posible emancipación. Como una concepción científica que posibilitó el paso del hombre abstracto al real" (Peña, Mena, Cardoso y Placeres, 2007).

Aquella corriente emplaza al ser humano, a los actores universitarios a cuestionar y/o validar: la misión y visión de la universidad, al rol de las IES frente al desarrollo de los pueblos, la pertinencia del currículo frente a la realidad, las metodologías de enseñanza. Se busca no perder de vista la problemática social, en la que se encuentran cientos y millones de seres humanos y que, probablemente, miran en la universidad y en la educación, una alternativa cierta para enfrentar sus adversidades.

Se requiere un enfoque trasformador, que se convierte en la razón histórica misma de la universidad, en cuyo seno las necesidades de los pueblos constituyen el motor que impulsa y que le da forma a la investigación y la docencia; se le proporciona una metodología participativa, consciente, recíproca y de compromiso, en la constante tarea de la democratización del conocimiento y del saber.

Parafraseado a Marx, (1844) diríamos que, "Si el hombre trabaja solo para sí, puede quizás ser un científico famoso, un gran sabio, un excelente poeta, pero jamás podrá ser un hombre perfecto y verdaderamente grande".

En consecuencia, la formación de los profesionales tiene en este enfoque, un aporte muy importante para superar el individualismo, más aún si en su interacción, se principaliza la corriente pedagógica socio crítica, que desde una noción social y científica, holística, pluralista e igualitaria, toma en cuenta a los actores universitarios y sociales, como los creadores de su propia realidad. Así, participan a través de su experiencia, para reafirmar el hecho de que los hombres y mujeres, “... son seres del quehacer, y por ello diferentes de los animales, seres del mero hacer. Los animales no admiran el mundo. Están inmersos en él. Por el contrario, los hombres como seres del quehacer emergen del mundo y objetivándolo pueden conocerlo y transformarlo con su trabajo (Freire, 1969).

Esto implica redimensionar a la vinculación con la sociedad, como una herramienta académica y técnica que impulsa la transformación de la realidad social y educativa, al mismo tiempo que, como la función, articula el quehacer de la vida universitaria; resignifica el conocimiento, las metodologías y sus relaciones en procura de formar profesionales comprometidos, con su historia, su patria y su pueblo.

1.2 Hacia un modelo de vinculación con la sociedad que articule las funciones sustantivas

En un escenario de importantes retos para la educación superior, la confluencia de factores legales y académicos determinados por la presencia de una nueva administración en la universidad en el año 2014, darán como resultado un escenario favorable para el desarrollo de la Vinculación con la Sociedad, luego de un largo proceso de desconcierto caracterizado por el olvido y hasta la persecución.

La existencia de una legislación válida para el desarrollo de la vinculación con la sociedad, constituía un aspecto formal del currículo, con pocas o ningunas atenciones en el plano administrativo y presupuestario, sin ninguna trascendencia en la política universitaria, apenas reconocida por docentes y estudiantes. Sus principales acciones se encontraban articuladas a las prácticas pre profesionales, con una serie de limitaciones conceptuales y técnicas, con pocas iniciativas y con contadas excepciones en las unidades académicas. "La última rueda del coche".

Lo señalado claramente se evidencia a través de los resultados presentados en la encuesta realizada a finales del año 2014 y comienzo del 2015. Como parte de la Red Ecuatoriana Universitaria de Vinculación con la Colectividad (REUVIC), dicha encuesta se realizó a 704 docentes y estudiantes de diferentes carreras de la UCE y cuyos resultados confirman la existencia de un sin número de problemas, que muestran la realidad aciaga, de los procesos de Vinculación o de Extensión. La 
mayor preocupación está en la falta de promoción y reconocimiento, seguido por la falta de recursos financieros y económicos, la falta de créditos para el currículo y la escasa prioridad de la institución por atender estos procesos como se puede observar en la siguiente gráfica.

PROBLEMAS QUE ENFRENTA SU UNIVERSIDAD EN LA GESTIÓN DEL PROCESO EXTENSIONISTA Y VINCULACIÓN CON LA
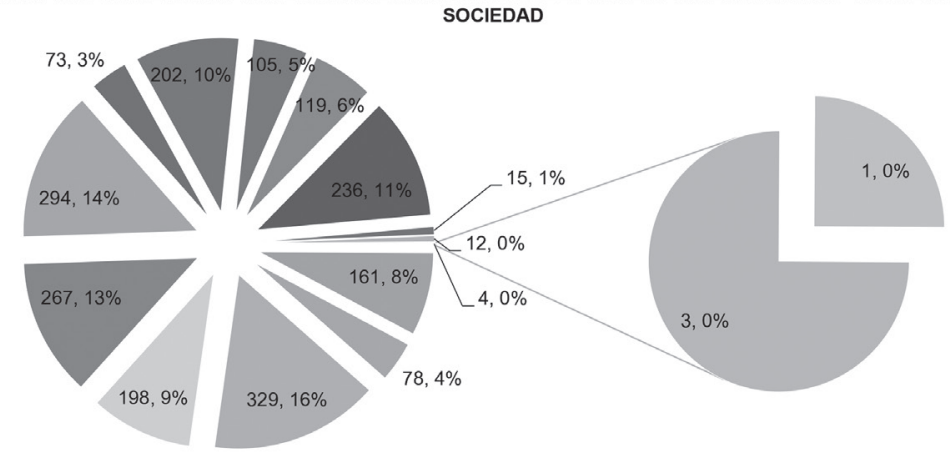

Desconocimiento de su importancia por parte de los directivos

Desconocimiento de su importancia por parte del claustro

- Falta de promoción y reconocimiento

-Falta de recursos financieros

- Falla de créditos para el curriculo

- Poca aceptabilidad por la población meta

- Poco tiempo para participar en las mismas

No contesta

- Falta de recursos humanos capacitados

- Falla de recursos económicos

- Inexistencia de lideres que impulsen la vinculación

- Poco dominio de las herramientas de trabajo necesarias

- No es prioridad de la institución

"Maltrato al estudiante universitario

"Falta de información

Figura 3. Encuesta de percepción, por REUVIC (2016)

En estas circunstancias y con la urgencia de orientar esta función sustantiva en la Universidad, se institucionaliza el Sistema de Vinculación con la Sociedad, como mecanismo que articula los procesos de las unidades académicas y que se propone impulsar a la luz de las necesidades sociales, programas y proyectos territoriales. Hay la participación directa de los actores comunitarios, si se toma como referencia los dominios académicos y los perfiles de las carreras participantes, en espera de la necesidad de la articulación con la docencia y la investigación científica.

El sistema aprobado en 2014 reconoce como líneas de acción a las prácticas pre profesionales, el trabajo comunitario, la educación continua, la asesoría, consultoría, movilidad y eventos como parte substancial de su macro proceso. Todo esto es ejecutado, monitoreado y evaluado por una estructura administrativa compuesta por un equipo central de coordinación y la presencia de coordinadores y responsables en las unidades académicas, cuya acción se encuentra determinada por el enfoque territorial y multidisciplinario. Al respecto, se señala que “... El territorio no es un espacio físico objetivamente existente, sino, una construcción social, es decir un conjunto de relaciones sociales que dan origen y a la vez expresan una identidad y un sentido de propósito compartido con múltiples agentes públicos y privados. Entendiéndose entonces por planificación territorial como un proceso permanente de análisis y evaluación del territorio que con enfoque sistémico y participativo permite formular objetivos a mediano y a largo plazo y desarrollar estrategias para alcanzarlos..." (Schejtman y Berdegué, 2004).

Dicho sistema institucionalizado, desde este enfoque, impulsa programas territoriales en el cantón Cayambe y Pedro Moncayo con la siguiente participación, como se refleja en la Tabla 1.

Tabla 1

Programas y proyectos de la UCE, por Secretaria Vinculación con la Sociedad UCE (2016)

\begin{tabular}{|c|c|c|c|c|}
\hline PROGRAMA & CONVENIO & $\begin{array}{l}\text { No. } \\
\text { FACULTADES }\end{array}$ & $\begin{array}{l}\text { No. } \\
\text { DOCENTES }\end{array}$ & $\begin{array}{l}\text { No. } \\
\text { Estudiantes }\end{array}$ \\
\hline $\begin{array}{l}\text { Programa de promoción de } \\
\text { vida saludable para la } \\
\text { población de Cayambe }\end{array}$ & UCE-GADIP & 12 & 115 & 1060 \\
\hline $\begin{array}{l}\text { Programa comunitario } \\
\text { para el impulso de la salud } \\
\text { integral de la población de } \\
\text { las Mancomunidades de } \\
\text { Gobiernos Parroquiales } \\
\text { rurales del norte de } \\
\text { Pichincha }\end{array}$ & UCE - MGPRNP & 8 & 28 & 260 \\
\hline $\begin{array}{l}\text { Programa Universidad del } \\
\text { Adulto y Adulto mayor. }\end{array}$ & UCE-GADIP & & 16 & 30 \\
\hline $\begin{array}{l}\text { Programa Universidad } \\
\text { para personas con } \\
\text { capacidades especiales. No } \\
\text { videntes. Federación de } \\
\text { Nacional de Ciegos. } \\
\text { FENCE. }\end{array}$ & UCE - FENCE & & 16 & 30 \\
\hline $\begin{array}{l}\text { Programa de extensión } \\
\text { cultural e identidad. }\end{array}$ & $\begin{array}{l}\text { UCE - } \\
\text { FUNDACIÓN } \\
\text { AZUCAR }\end{array}$ & 1 & 12 & 150 \\
\hline
\end{tabular}


El reto en estas circunstancias constituye la urgencia para edificar un proyecto educativo, que articule las líneas de investigación, con las funciones sustantivas de la universidad. El eje central de desarrollo es la vinculación con la sociedad que finalmente permita constituir:

- Un currículo contextualizado con la realidad del entorno social y de su trascendencia histórica.

- Una estructura curricular que integre y visibilice la interculturalidad, el género y la inclusión.

- Una estructura curricular que incluya de forma secuencial e integral la investigación formativa y generativa, entre otros aspectos.

Como parte de un proceso de construcción colectiva, se pretende articular de forma dialéctica y secuencial a la docencia, como proceso de participación equitativa, que proporcione al estudiante herramientas esenciales para el desarrollo de capacidades y valores en escenarios comunitarios e interdisciplinarios como entornos reales de aprendizaje. En dichos espacios, la investigación constituye un instrumento pedagógico y didáctico para la deconstrucción de saberes. De esta forma, se aprende a aprender mediante la identificación y el tratamiento a la problemática social vinculada a su profesión, mediante la praxis, en los programas y proyectos de vinculación con la sociedad, en cada uno de las comunidades territoriales intervenidas.

Si colocamos al centro de la acción académica, como eje de desarrollo a la vinculación con la sociedad, es posible aportar de forma significativa para que se consolide una educación superior, democrática, abierta, colaborativa, colectiva, crítica, en la cual, el proceso de enseñar - aprender solvente circunstancias de la vida. Así se promueven puentes metodológicos que sustentan la docencia, investigación y vinculación en las comunidades y el aula, como estructuras sociales que se estimulan, dinamizan e impulsan cada uno de ellos, mediados por el compromiso comunitario entre académicos y organizaciones sociales.

Esta interacción trasciende a lo inter no y externo de las IES, fundamentalmente en la memoria colectiva de sus actores, como requisito para abandonar la transferencia de conocimiento. Esto favorece el intercambio de saberes,

\section{PROCESOS DE PERTINENCIA DE LA VINCULACIÓN CON LA SOCIEDAD}

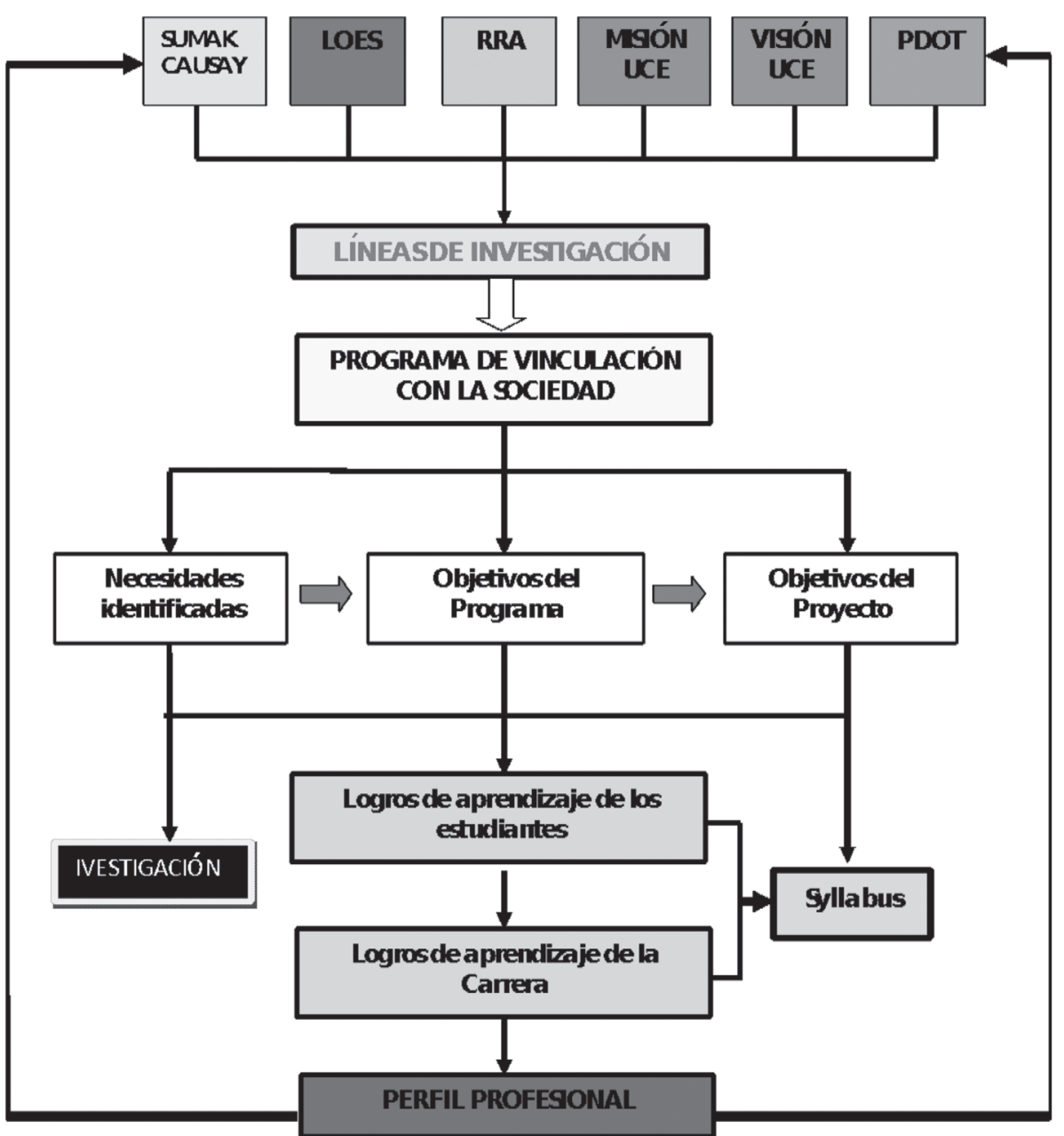

Figura 4. Proceso de pertinencia de la Vinculación con la Sociedad, por Sistema de Vinculación UCE (2016) 
mediante un proceso que permite recrear o innovar el conjunto de saberes, con permanente movilización de los actores y el uso del conocimiento de una manera colaborativa. Al respecto, Hall, (2009) plantea que "un cambio modesto en la mirada institucional, junto con un conjunto de estructuras de colaboración imaginativas, podrían tener un impacto muy visible en problemáticas sociales de hoy, como la gente sin techo, los problemas alimentarios o la salud y la educación de los pueblos aborígenes" (p. 39).

En términos similares, Laing y Maddison, (2007: p. 13) destacan que "si insistimos en la idea de ampliar la participación en el marco de la educación superior, de ensanchar el acceso a los recursos que tienen las universidades, más allá del acceso a los cursos, nos encontramos con diferentes escenarios, sobre todo si introducimos un cambio sustancial en nuestro ángulo de visión".

Es una visión nueva, renovada, contextual y transformadora que supera la aspiración o interés fundamental de la docencia y la investigación tradicional, alejada de las demandas sociales y de uso exclusivo de los expertos, en función básicamente de calificar la mano de obra para los procesos de producción.

\section{Referencias}

Batista de los Ríos, D. (2011). Liderazgo de la universidad en la sociedad de conocimiento desde la dimensión extensionista. Revista Conexão $U E P G$, ISSN-e 2238-7315, Vol. 7, №. 2, (pp. 152-161).

Freire, P. (1969). El acto de leer y el proceso de liberación (pp.150). Montevideo, México: Tierra Nueva.
Hall, B. (2009). The Funding and Development of Community-University Research Partnerships in Canada. Ottawa, Cánada: SSHRC.

Laing, S., Maddison, E. (2007). Estructuras de investigación colaborativa comunidad universidad: aproximación a su posible impacto, Rizoma freireano. Instituto Paulo Freire de España. Vol. 9.

MarX, K. (1844). Manuscritos económicos y filosóficos de 1844. Primera Edición. Marx/Engels Gesamtausgabe. Авт. 1, BD. 3

Pena, G., Mena, M. y Cardoso, J., Placeres, M. (2007). La Teoría Marxista sobre el Humanismo. Rev Hum Med, Vol.7, N 2 .

Schejtman, A y Berdegué, J. (2004). Desarrollo territorial rural. Documento elaborado para la División América Latina y el Caribe del Fondo Internacional de Desarrollo Agrícola (FIDA) y el Departamento de Desarrollo Sustentable del Banco Interamericano de Desarrollo (BID). Santiago, Chile.

\section{Netgrafía}

Wilson, R. (2012). Influencia de la concepción curricular de la asignatura de diseño arquitectónico, en el proceso de formación en los estudiantes y propuesta de una actualización curricular, en la facultad de arquitectura y urbanismo de la UCE. Recuperado de www.dspace.uce.edu.ec:8080/ bitstream/25000/750/1/T-UCE-0010-204.pdf 